\title{
Abordagens e recursos didáticos voltados ao processo de ensino-aprendizagem de Geometria focados em alunos cegos - uma Revisão Sistemática da Literatura
}

\author{
Approaches and Didactic Resources for the Teaching-learning Process of Geometry
} Focused on Blind Students - a Systematic Literature Review

ANDRADE, Rebeca Medeiros de; Mestranda; PGDesign - UFRGS

rebeca.andrade@ufrgs.br

SILVA, Régio Pierre da; Doutor; PGDesign - UFRGS

regio@ufrgs.br

SILVA, Tânia Luísa Koltermann da; Doutora; PGDesign - UFRGS

tania.koltermann@ufrgs.br

TEIXEIRA, Fábio Gonçalves; Doutor; PGDesign - UFRGS

fabiogt@ufrgs.br

\section{Resumo}

O objetivo central desse trabalho foi analisar publicações científicas, com o propósito de identificar as possíveis abordagens, métodos, processos, técnicas e tecnologias relacionadas ao ensino e aprendizagem de geometria focados em alunos cegos, em fase escolar. Para isso, foi realizada uma revisão sistemática da literatura, que selecionou quatro trabalhos, de acordo com os procedimentos de busca, inclusão e exclusão estabelecidos. Os trabalhos selecionados forneceram informações quanto a questão da inclusão de pessoas com deficiência no contexto escolar, adaptação de conteúdos como apoio ao ensino e aprendizagem, métodos e ferramentas que possibilitam ao estudante cego a construção de modelos mentais por meio de exploração tátil. A síntese dos dados levantados trouxe a perspectiva de diferentes áreas da pesquisa no âmbito do ensino de geometria, com foco no atendimento das necessidades educacionais especiais de alunos com deficiência visual.

Palavras Chave: Geometria; Tecnologia Assistiva; Produção de Conhecimento.

\begin{abstract}
The aim of this study was to analyze scientific publications in order to identify possible approaches, methods, processes, techniques and technologies related to the process of teaching and learning of geometry focused on blind students. Thus, a systematic literature review was conducted, which selected four papers, according to the established search, inclusion and exclusion procedures. The selected papers provided information about the inclusion of people with disabilities in the school context, adaptation of content to support teaching and learning, methods and tools that enable the blind student to construct mental models through tactile exploration. The synthesis of the data gathered brought the perspective of different areas of research in the field of geometry teaching,
\end{abstract}


with a focus on meeting the special educational needs of students with visual impairments.

Keywords: Geometry; Assistive Technology; Knowledge Production.

\section{Introdução}

O objetivo da presente Revisão Sistemática da Literatura foi levantar e analisar dados referentes ao cenário atual da situação de estudantes cegos no que diz respeito ao ensino e aprendizagem de geometria. A análise foi feita com base em artigos científicos nacionais e internacionais publicados no período entre 2010 e 2017, com o objetivo de levantar evidências sobre o ensino de geometria a indivíduos cegos, pesquisar a possível utilização de métodos, ferramentas, materiais e/ou dispositivos que possibilitem ao estudante cego a construção de modelos mentais por meio de exploração da percepção tátil, e ainda observar as prováveis relações existentes entre inclusão da Pessoa com Deficiência (PCD) por meio da adaptação de conteúdos como apoio à aprendizagem e o processo de Design.

Como apontado por Dresch, Lacerda e Júnior (2015), a Revisão Sistemática da Literatura é uma etapa de fundamental importância no desenvolvimento de pesquisas científicas, pois identificam e apresentam pesquisas pertinentes que foram feitas em determinada área, possibilitando ao pesquisador ter uma visão mais ampla do cenário no qual pretende trabalhar, pesquisar.

Foram definidas três fases para a execução da presente Revisão Sistemática da Literatura. Fase inicial, que possui 4 etapas: definição dos objetivos e questões de pesquisa, definição de fontes de busca, definição da string de busca e definição de critérios de inclusão e exclusão e de qualidade. Fase de busca, inclusão e exclusão: consiste na utilização da string para busca, e os critérios de inclusão e exclusão são aplicados para seleção dos artigos científicos. Fase de análise e síntese: momento no qual todo o material selecionado após a aplicação de todos os filtros é analisado tendo como base, inclusive, os critérios de qualidade definidos no item 2.3.

Figura 1 - Fases definidas para a execução da Revisão Sistemática da Literatura

\begin{tabular}{|c|c|c|c|}
\hline $\begin{array}{l}\text { FASE } \\
\text { INICIAL }\end{array}$ & $\begin{array}{r}\text { FASE DE } \\
\text { INCLUSÃO E }\end{array}$ & $\begin{array}{l}\text { BUSCA, } \\
\text { EXCLUSÃO }\end{array}$ & $\begin{array}{c}\text { FASE DE } \\
\text { ANÁLISE E SİNTESE }\end{array}$ \\
\hline \multirow{6}{*}{ 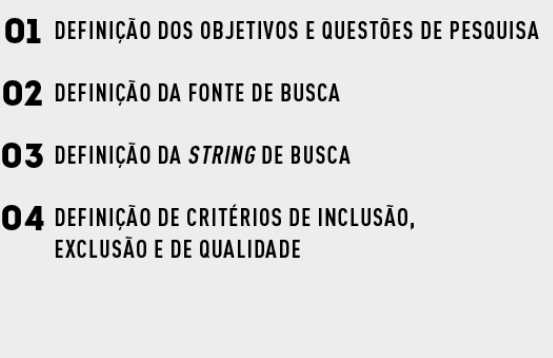 } & BUSCA POR STRING & 75 TRABALHOS & \multirow{2}{*}{$\frac{\text { APLICAÇÃO DOS CRITÉRIOS DE QUALIDADE }}{\text { ANÁLISE DO MATERIAL COLETADO }}$} \\
\hline & ARTIGOS CIENTÍFICOS & 63 TRABALHOS & \\
\hline & PERÍODO 2010 - 2017 & 23 TRABALHOS & \multirow[t]{4}{*}{ SÍNTESE } \\
\hline & ANÁLISE dE TÍTULO & 12 TRABALHOS & \\
\hline & LEITURA DE ABSTRACT & 07 TRABALHOS & \\
\hline & LEITURA DE ARTIGO & 04 TRABALHOS & \\
\hline
\end{tabular}

Fonte: a autora

\section{Contexto}

Indivíduos cegos possuem capacidades de desenvolvimento tanto quanto indivíduos videntes, diferindo apenas na velocidade com a qual esse processo se dá (ARGYROPOULOS, 2002). 
Como exemplo, pode ser citado o fato de que habilidades cognitivas se desenvolvem num ritmo mais lento ou de modo diferente em crianças cegas em comparação a crianças videntes, como apontado por Warren (1984), mas o desenvolvimento se dá de qualquer forma, desde que seja estimulado.

Com base nesses fatos, a necessidade de se realizar esse estudo se dá pelo fato de que é importante identificar os avanços científicos em relação ao ensino focado na criança cega. A necessidade de se fazer uma Revisão Sistemática advém principalmente da necessidade de obter uma visão abrangente da área de pesquisa, considerando estudos e pesquisas já realizadas para, dessa forma, evitar o desenvolvimento de trabalhos irrelevantes, trabalhos que não sejam realmente necessários ou que sejam antiéticos (DRESCH; LACERDA; JÚNIOR, 2015).

\subsection{Objetivo e questões de pesquisa}

O objetivo central da presente Revisão é analisar evidências extraídas de publicações científicas, com o propósito de identificar as possíveis abordagens, os métodos ou processos, técnicas e tecnologias relacionadas ao ensino e aprendizagem de geometria do ponto de vista das necessidades reais demandadas por alunos cegos, no contexto acadêmico da fase escolar.

Com a intenção de clarificar conceitos e nortear a fase de análise e síntese do material coletado, foram definidas três questões de pesquisa:

- $\quad$ Como foi abordada a questão da inclusão da Pessoa com Deficiência (PcD) por meio da adaptação de conteúdos como apoio à aprendizagem?

- Quais são os métodos/ferramentas/materiais/dispositivos que podem ser utilizados para possibilitar ao estudante cego a construção de modelos mentais por meio de exploração da percepção tátil?

- $\quad$ Existe a proposição de uma relação entre processo de ensino por meio de adaptação de conteúdo e processo de Design?

\subsection{Estratégias utilizadas e fontes de pesquisa}

Antes de iniciar o processo de busca propriamente dito, foram testadas algumas opções de strings relacionadas ao objeto de estudo, com o intuito de observar como seria o retorno de algumas combinações de expressões gerais de busca. Após alguns testes utilizando strings complexas e outras mais simples/curtas, ficou definido o uso da uma string de busca que traz os termos organizados em hierarquia de relevância para a pesquisa e que estão conectados por meio de operadores lógicos: ("blind students" AND mathematics AND geometry).

Em relação às fontes de pesquisa, num primeiro momento foi selecionado o banco de dados Scopus (https://www.scopus.com), pois o mesmo é reconhecido por ser uma das maiores fontes de trabalhos e literaturas científicas. O fato de a busca (utilizando a string definida) ter retornado apenas três resultados fez com que novas buscas fossem feitas em outras fontes. Optou-se então por dar prosseguimento à pesquisa no Portal de Periódicos da Coordenação de Aperfeiçoamento de Pessoal de Nível Superior (Capes) (http://www.periodicos.capes.gov.br), utilizando-se a mesma string de busca utilizada no primeiro momento. 
Figura 2 - String de busca que retornou apenas 3 resultados

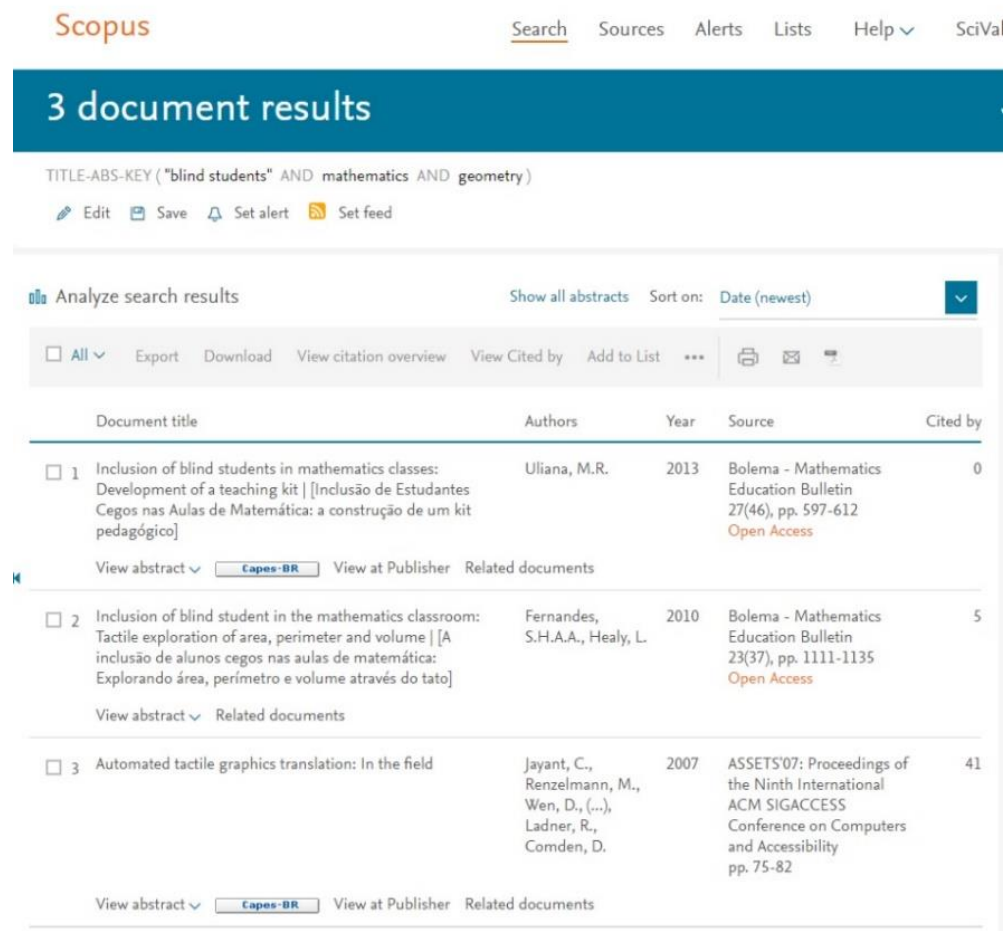

Fonte: print screen da busca feita na Base Scopus em junho de 2017

\subsection{Critérios adotados para a inclusão de fontes}

Os seguintes critérios foram adotados para inclusão de fontes: a) ser artigo científico, b) ter sido publicado no intervalo entre 2010 e 2017, c) estar relacionado à área de pesquisa do presente trabalho, d) o idioma ser Português ou Inglês e e) ter o texto completo disponível (open access).

Para exclusão de fontes, foram adotados os seguintes critérios: a) não ser um artigo científico, b) ter sido publicado antes de 2010 , c) não possuir relação com a área de pesquisa do presente trabalho e d) não estar disponível para acesso completo (open access).

Para atribuição de valor (em termos de qualidade e relevância) às publicações selecionadas, serão considerados os seguintes critérios de qualidade:

- $\quad$ (CR1) Aborda a questão da inclusão da Pessoa com Deficiência (PcD) por meio da adaptação de conteúdos como apoio à aprendizagem.

- $\quad$ (CR2) Faz alusão ao ensino de geometria.

- (CR3) Apresenta algum método/ferramenta/material/dispositivo que possibilite ao estudante cego a construção de modelos mentais por meio de exploração da percepção tátil.

- $\quad$ (CR4) Estabelece algum tipo de relação com o processo de Design.

\section{Busca, inclusão e exclusão}

Os procedimentos de busca, inclusão e exclusão de publicações se dá de forma quase que 
simultânea em alguns momentos. No primeiro momento, a busca na base de dados, a string que foi definida é utilizada, e somando-se a ela, são usadas as ferramentas de filtragem disponíveis no banco de dados. Para a busca que foi feita, foram usados os filtros de período (critério de inclusão b) e tipo de trabalho (critério de inclusão a). Dessa forma foi possível ter acesso ao primeiro grande grupo de trabalhos com potencial de serem usados na presente Revisão, e nesse grande grupo inicial foram aplicados os critérios de inclusão/exclusão.

A busca por string retornou 75 resultados, destes foram excluídos 12 trabalhos por não serem artigos científicos (critério de exclusão a). Dos 63 artigos científicos que ficaram, foram excluídos 40 por não terem sido publicados no período entre 2010 e 2017 (critério de exclusão b). Dos 23 artigos que correspondiam ao período pré-estabelecido, apenas 12 apresentavam títulos com algum tipo de relação ao foco de interesse do presente trabalho (critério de exclusão c). Desses 12 artigos, apenas 7 foram selecionados após a leitura do abstract (critério de inclusão c), pois apresentavam conteúdo com provável relevância para o desenvolvimento da Revisão Sistemática. Desses 7 trabalhos, apenas 4 foram selecionados para leitura (critério de inclusão e), pois os outros 3 não estavam com texto completo disponível (open access), que era um dos critérios definidos para inclusão/exclusão. Na Tabela 1 estão relacionados os trabalhos selecionados, seus respectivos anos de publicação, seus respectivos autores e a fonte do trabalho.

Tabela 1 - Artigos selecionados para leitura

\begin{tabular}{l|c}
\multicolumn{1}{c}{ TíTULO DO ARTIGO } & AUTOR \\
\hline $\begin{array}{l}\text { Faptic cues as a utility to perceive and recognise geometry. (2013) } \\
\text { Hvannberg, E.T. \& } \\
\text { Hafsteinsson, H. }\end{array}$ & $\begin{array}{c}\text { Shimomura, Y., Springer } \\
\text { Hin E }\end{array}$ \\
\hline $\begin{array}{l}\text { Inclusão de Estudantes Cegos nas Aulas de Matemática: a } \\
\text { construção de um kit pedagógico. (2013) }\end{array}$ & Uliana \\
\hline $\begin{array}{l}\text { An exploratory study of visually impaired students' perceptions of } \\
\text { inclusive mathematics education. (2015) }\end{array}$ & Bayram \\
\hline $\begin{array}{l}\text { Technologies used in the study of advanced mathematics by } \\
\text { students who are visually impaired in classrooms: teachers' } \\
\text { perspectives. (2015) }\end{array}$ & SAGE \\
\hline
\end{tabular}

Fonte: a autora

Com a finalidade de atribuição de valor (em termos de qualidade e relevância para a Revisão Sistemática), cada um dos quatro critérios de qualidade será avaliado tendo como base uma pontuação simbólica, na qual 10 corresponde ao fato de o trabalho atender completamente ao critério de qualidade observado, 5 ao atendimento incompleto, abordagem superficial ou indireta do critério e 0 será atribuído no caso de o critério não ter sido atendido (ou sequer ter sido citado).

Na Tabela 2 estão apresentados os valores que foram atribuídos a cada publicação, com base nos critérios de qualidade no item 2.3 e conforme explicado acima a respeito da atribuição de valores. 
Tabela 2 - Avaliação de trabalhos por critérios de qualidade, segundo item 2.3

\begin{tabular}{|c|c|c|c|c|c|c|}
\hline AUTOR & TÍTULO DO ARTIGO & CR1 & CR2 & CR3 & CR4 & TOTAL \\
\hline Bayram & $\begin{array}{l}\text { An exploratory study of visually impaired students' } \\
\text { perceptions of inclusive mathematics education. } \\
\text { (2015) }\end{array}$ & 10 & 0 & 5 & 0 & 15 \\
\hline DePountis & $\begin{array}{l}\text { Technologies used in the study of advanced } \\
\text { mathematics by students who are visually } \\
\text { impaired in classrooms: teachers' perspectives. } \\
\text { (2015) }\end{array}$ & 10 & 5 & 0 & 0 & 15 \\
\hline $\begin{array}{l}\text { Shimomura, Y., } \\
\text { Hvannberg, E.T. \& } \\
\text { Hafsteinsson, H. }\end{array}$ & $\begin{array}{l}\text { Haptic cues as a utility to perceive and recognise } \\
\text { geometry. (2013) }\end{array}$ & 10 & 10 & 10 & 10 & 40 \\
\hline Uliana & $\begin{array}{l}\text { Inclusão de Estudantes Cegos nas Aulas de } \\
\text { Matemática: a construção de um kit pedagógico. } \\
\text { (2013) }\end{array}$ & 10 & 10 & 10 & 0 & 30 \\
\hline
\end{tabular}

Fonte: a autora.

\section{Análise e síntese dos trabalhos coletados}

Os trabalhos selecionados forneceram importantes informações a respeito do tema de interesse da presente Revisão da Literatura. A seguir serão apresentadas as contribuições obtidas de cada trabalho, referentes aos critérios de qualidade, porém antes será apresentado um breve panorama dos assuntos abordados por cada um dos autores selecionados.

Em seu trabalho, Bayram et al. (2015) se propuseram a explorar os desafios enfrentados por indivíduos deficientes visuais enquanto estudantes de Matemática, do ensino médio, em salas de aula inclusivas. A pesquisa foi do tipo qualitativa e foi feita em escolas na Turquia (contexto geográfico, social e cultural dos pesquisadores), os dados foram obtidos por meio de observação de uso de métodos/ferramentas de ensino/aprendizagem usados em sala de aula, e também por meio de entrevistas. Por meio das informações obtidas através das entrevistas, percebeu-se que apesar de muitas das demandas sociais em relação à inclusão dos indivíduos com deficiência serem atendidas, demandas relacionadas à educação não apresentavam os mesmos resultados. Assim os pesquisadores levantaram a hipótese de que havia uma provável postura negativa dos professores de matemática em relação à educação inclusiva, ocasionando barreiras ao processo de aprendizagem dos alunos com deficiência.

O trabalho de DePountis et al. (2015) foi conduzido à luz da necessidade de os professores de matemática se manterem constantemente atualizados em relação às ferramentas de ensino que podem auxiliar no processo de aprendizagem de alunos com deficiência visual (sendo eles cegos ou com baixa acuidade visual). Os autores apontam o fato de nos últimos 30 anos ter havido um verdadeiro boom tecnológico, que produziu uma abundância de ferramentas de real utilidade que auxiliam tanto alunos no que diz respeito ao aprendizado, quanto professores no que diz respeito ao ensino. Uma das ferramentas mais citadas no trabalho são as leitoras de Braille, e os autores ressaltam a importância de os professores se manterem atualizados em relação às potencialidades funcionais dessa ferramenta, suas variações, adaptações e possíveis conexões com outros dispositivos, como impressoras, por exemplo.

Shimomura, Hvannberg e Hafsteinsson (2013) apresentam em seu trabalho um riquíssimo conteúdo que aborda de forma extensiva a utilização de haptics em dispositivos desenvolvidos 
para público cego (ou de baixa acuidade visual). Os autores abordam a importância da combinação de recursos para potencializar a percepção por parte de indivíduos cegos, podendo ser recursos visuais, auditivos, táteis ou a combinação destes. E no que diz respeito ao desenvolvimento de interfaces, a combinação desses recursos como sendo inputs e outputs, o termo utilizado é interface multimodal. Os autores ainda apontam que o forte incentivo para a utilização de haptics é o fato de que o aprendizado ativo é mais eficiente do que o aprendizado passivo, já que a possibilidade de experienciar por meio do tato se mostra mais eficiente do que o simples ato de assistir algo.

Já no trabalho da pesquisadora brasileira Uliana (2013), percebe-se que a motivação para pesquisa se deu por uma demanda pessoal, visto que a mesma dava aulas de matemática e percebeu a necessidade de criar um kit de material pedagógico que auxiliasse no processo de ensino/aprendizagem dos conteúdos da geometria plana dos estudantes cegos de ensino fundamental. Quando a pesquisadora percebeu essa necessidade, ela não tinha conhecimento da existência do Multiplano, que é um rico material desenvolvido por Ferronato (2002), que possibilita aos alunos cegos (e aos típicos também) a construção e análise de gráficos e funções (entre outras possibilidades ligadas ao estudo da Matemática). A proposta da professora foi desenvolver uma ferramenta que proporcionasse ao aluno cego a mesma ação que o aluno vidente realiza usando papel, lápis e régua, mesmo que para isso fossem usados outros materiais, sendo eles: campo magnético com ímãs, no qual o aluno tem autonomia para localizar pontos cartesianos (com qualquer coordenada), esboçar e analisar gráficos, esboçar formas geométricas planas com tamanhos e formatos distintos, dentre outras possibilidades.

\subsection{Como foi abordada a questão da inclusão da Pessoa com Deficiência (PcD) por meio da adaptação de conteúdos como apoio à aprendizagem?}

Dos quatro critérios de qualidade estabelecidos, este foi o único que foi amplamente abordado nos artigos selecionados para leitura. As abordagens possuem focos distintos, dado que os artigos pesquisam áreas também distintas dentro do universo do ensino/aprendizagem dos conteúdos da Matemática focados nos estudantes cegos e/ou com baixa acuidade visual.

Bayram et al. (2015) se apoiam no trabalho de Bartell e Meyer (2008) e apontam que a igualdade em salas de aula de Matemática significa atender às necessidades dos alunos através da criação de um ambiente mais justo, no qual todos os alunos, cegos ou videntes, possuam igualdade de oportunidade para explorar seu potencial. Afirmam também que a igualdade em sala de aula requer uma ação de ensino efetiva por parte dos professores, e conforme apontamentos de Allexsaht-Snider e Hart (2001), essa é a forma de minimizar a discrepância entre alunos com diferentes tipos e graus de necessidades enquanto aprendem Matemática num ambiente verdadeiramente produtivo.

DePountis et al. (2015) afirmam que o ensino de alta qualidade é aquele que incorpora ferramentas que ajudem os alunos (que possuam ou não deficiência visual) a ter um melhor acesso e entendimento da Matemática, considerando suas capacidades e os direcionando para o melhor que podem fazer. Apontam ainda que no caso de professores que possuem algum aluno com deficiência visual, a existência e uso de tecnologia nas aulas não se trata de uma questão opcional, mas necessária. 
Apoiados em resultados de pesquisas na área de educação Matemática, Shimomura, Hvannberg e Hafsteinsson (2013) apontam para a necessidade de criação de novos modelos de ensino de geometria. Os autores afirmam que deveria ser dada maior ênfase na exploração de objetos tridimensionais como uma forma de complementar recursos tecnológicos computacionais, pois se reconhece que a pesquisa em geometria tridimensional no ambiente de realidade virtual é de grande necessidade para auxiliar o desenvolvimento das habilidades espaciais dos alunos.

O trabalho da pesquisadora Uliana (2013) consistiu na criação de um material de apoio ao ensino/aprendizagem de geometria plana, que não exigisse do professor uma mudança radical em sua maneira de trabalhar conteúdos matemáticos e que também não o forçasse a investir muito tempo de sua aula dando atendimento individual aos alunos com deficiência visual. Apoiada em alguns conceitos abordados na pesquisa de Ferronato (2002), a pesquisadora acredita que o uso frequente de materiais adaptados e/ou desenvolvidos com foco no aluno deficiente visual e que possibilitem ao mesmo ter acesso aos mesmos conteúdos, porém através do tato, já significam uma forma de inclusão.

\subsection{Quais são os métodos/ferramentas/materiais/dispositivos que podem ser utilizados para possibilitar ao estudante cego a construção de modelos mentais por meio de exploração da percepção tátil?}

Da perspectiva de Bayram et al. (2015) no que diz respeito ao uso de materiais diferenciados no processo de aprendizagem de Matemática, os pesquisadores afirmam que diferentes tipos de recursos táteis podem ser utilizados para fazer com que haja uma compreensão mais profunda dos conceitos matemáticos, porém o ponto em comum entre esses diferentes recursos deve ser a capacidade de permitir ao estudante aprender por meio de seu próprio entendimento e capacidade de assimilação, mas garantindo que haverá apoio caso eles sintam necessidade.

Os pesquisadores apontam ainda que os materiais de maior utilidade destinados a indivíduos com deficiência visual são aqueles que possuem pouco ou nenhum componente visual e aqueles que possuem suporte por meio de áudio. Apesar de fornecerem essa perspectiva em relação ao uso de materiais e recursos que podem ser utilizados para possibilitar aos estudantes cegos a construção de modelos mentais, o assunto não é amplamente discutido e não são apresentados modelos reais utilizados ou que são propostos pelos autores, os menos apontam apenas o que julgam ser o mais adequado.

Shimomura, Hvannberg e Hafsteinsson (2013) apontam para o fato de que através do uso de diferentes tecnologias, pesquisadores vêm explorando e estudando as habilidades de indivíduos cegos, há mais de uma década, no reconhecimento de objetos tridimensionais. Isso aponta para a realidade de que pode ser pesquisado como e onde esse potencial pode ser melhor empregado, e também como os outros sentidos humanos podem ser explorados com a intenção de possibilitar a interação, de forma efetiva, de indivíduos cegos com computadores. Os pesquisadores afirmam ainda que estudar até que ponto os indivíduos cegos estão aptos para conceber modelos mentais de objetos geométricos de baixa complexidade e suas propriedades geométricas, e estudar ainda a manipulação de dispositivos em ambientes virtuais podem auxiliar no desenvolvimento de aplicativos destinados a estudantes cegos. 
Conforme aponta Uliana (2013), a correta utilização de materiais concretos em aulas de Matemática (que se trata de uma disciplina que trabalha muito a questão de formas e padrões) representa uma oportunidade de o aluno cego ou de baixa acuidade visual ter acesso às experiências comuns, por meio do tato, que fazem com que o mesmo amplie sua bagagem de conhecimento. A utilização de materiais concretos reduz a possibilidade de abstração, o que se torna um fator positivo quando se trata de situações de ensino/aprendizagem de Matemática em contextos que envolvam indivíduos com deficiência visual.

A pesquisadora apresenta a criação e desenvolvimento de um material pedagógico idealizado com a intenção de ensinar sobre construção e análise de gráficos de funções do tipo polinomial de primeiro e segundo grau a um aluno cego, para o qual dava aulas em uma escola regular na cidade de Cacoal - RO, em 2006. O material, inicialmente nomeado Plano Cartesiano de Metal, é um kit pedagógico que possui:

[...] uma placa de metal com manta magnética quadriculada em uma das faces, eixos $\mathrm{x}$ e y de ímã com numeração em Braille, formas geométricas planas em EVA com manta magnética em uma das faces, pinos de ímãs, pedaços de arame flexível e pedaços de raios de bicicleta de tamanhos variados (ULIANA, 2013, p. 597).

O kit possibilita ao aluno cego, através do tato, realizar diferentes atividades matemáticas que envolvam gráficos, funções polinomiais e até mesmo figuras geométricas planas.

\subsection{Existe a proposição de uma relação entre processo de ensino por meio de adaptação de conteúdo e processo de Design?}

O único trabalho que faz alusão a processos de Design é o dos pesquisadores Shimomura, Hvannberg e Hafsteinsson (2013). Os outros trabalhos abordam mais a relação entre alunos e professores, a necessidade de adaptação de conteúdos como estratégia de ensinagem que favorece a inclusão em sala de aula, utilização de recursos tecnológicos como apoio ao processo de ensino/aprendizagem.

A respeito do trabalho desenvolvido por Shimomura, Hvannberg e Hafsteinsson (2013), o processo de Design foi explorado na pesquisa em usabilidade, o que incluiu testes de um protótipo de aplicativo com usuários cegos, testes esses que indicaram presença de guidelines para interação de haptics. O objetivo central definido a partir das guidelines foi o desenvolvimento de produtos com design de fácil utilização, eficiente, de fácil aprendizagem e de aceitação positiva por parte do usuário. No âmbito das guidelines, os pesquisadores classificaram a interação por meio de haptics de acordo com cinco aspectos: objetos virtuais, navegação com ampla visibilidade do ambiente virtual, contexto de objetos virtuais, módulos e aprendizagem de programação. Os autores ainda dedicam uma seção do seu trabalho a abordagem de conceitos referentes às suas pesquisas no que diz respeito ao Design para Experiência.

\section{Considerações Finais}

O processo de Design foi, sem dúvida, o tema com menor incidência de abordagem nos trabalhos selecionados. Se por um lado surge a indagação se o tema pode ser relativamente novo e pouco abordado no meio científico, por outro lado supõe-se que seja necessária a definição de uma nova string de busca, na qual o termo Design apareça. Provavelmente essa será a forma mais 
efetiva de obter resultados de busca que contemplem essa esfera de conhecimento, já que o processo de Design no desenvolvimento de ferramentas de apoio ao ensino de geometria é o foco de pesquisa do presente trabalho.

Com relação aos outros assuntos de interesse da pesquisa, acredita-se que os resultados de busca foram bem relevantes, pois além de trazer a perspectiva de diferentes áreas de pesquisa no âmbito do ensino de geometria, ainda fornece conteúdo atualizado sobre o tema, já que as pesquisas selecionadas para leitura foram publicadas em 2013 e 2015, de acordo com o critério de inclusão/exclusão b) artigos científicos publicados no período entre 2010 e 2017.

O fato de a utilização de string de busca e aplicação de filtros ter retornado trabalhos com enfoques distintos a respeito do mesmo tema, denota a riqueza dos conteúdos científicos que podem ser explorados. A presente Revisão possibilitou o acesso ao trabalho desenvolvido pela professora e pesquisadora Uliana (2013), que consiste na criação de um modelo de ensino/aprendizagem desenvolvido de forma artesanal, focado no atendimento de necessidades de alunos deficientes visuais. Possibilitou também o acesso a conteúdo focado na utilização de modelos de ensino/aprendizagem com maior apelo e exploração tecnológica de dispositivos sensoriais táteis, como apresentado pelos pesquisadores Shimomura, Hvannberg e Hafsteinsson (2013). Possibilitou ainda o acesso ao trabalho de Bayram et al. (2015), que apontam para a importância do desenvolvimento de uma nova mentalidade e postura positiva por parte dos professores que lidam com alunos com deficiência, no que diz respeito a promoção de uma educação mais inclusiva. E, por fim, o acesso ao trabalho de DePountis et al. (2015), que é uma rica contribuição em relação à reafirmação da necessidade de elevação da qualidade das estratégias de ensinagem por parte de professores e pontuam a real necessidade de se fazer uso constante de tecnologia assistiva em sala de aula.

\section{Referências}

ALLEXSAHT-SNIDER, M., \& HART, L. E. 'Mathematics for all': How do we get there? Theory into Practice, 2001. 40, p. 93-101.

ARGYROPOULOS, V. S.; ARGYROPOULOS, V. S. Tactual shape perception in relation to the understanding of geometrical concepts by blind students. British Journal of Visual Impairment, 1 jan. 2002. v. 20, n. 1, p. 7-16.

Bartell, T. G., \& Meyer, M. Y. Addressing the equity principle in the mathematics classroom. Mathematics Teacher, 2008. 101, p. 604-608.

BAYRAM, G. i. et al. An exploratory study of visually impaired students' perceptions of inclusive mathematics education. British Journal of Visual Impairment, 2015. v. 33, n. 3, p. 212-219.

DEPOUNTIS, V. M. et al. Technologies used in the study of advanced mathematics by students who are visually impaired in classrooms: teachers' perspectives. Journal of Visual Impairment \& Blindness, 2015. v. 109, n. 4, p. 265-278.

DRESCH, A.; LACERDA, D. P.; JÚNIOR, J. A. V. A. Design Science Research: Método de Pesquisa para Avanço da Ciência e Tecnologia. Porto Alegre: Bookman Editora, 2015.

FERRONATO, R. A construção de um instrumento de inclusão no ensino da matemática. $124 f$. Dissertação (Mestrado em Engenharia de Produção) - Universidade Federal de Santa Catarina, 
Florianópolis, 2002.

SHIMOMURA, Y.; HVANNBERG, E. T.; HAFSTEINSSON, H. Haptic cues as a utility to perceive and recognise geometry. Universal Access in the Information Society, 2013. v. 12, n. 2, p. 125-142.

ULIANA, M. R. Inclusão de Estudantes Cegos nas Aulas de Matemática: a construção de um kit pedagógico. Bolema: Boletim de Educação Matemática, 2013. v. 27, n. 46, p. 597-612.

WARREN, David H. Blindness and Early Childhood Development. New York: American Foundation for the Blind. 384 pages. 1984. 